\title{
Pion Production from Proton Synchrotron Radiation under Strong Magnetic Field in Relativistic Quantum Approach
}

\author{
Tomoyuki Maruyama1,3,a , Myung-Ki Cheoun², Toshitaka Kajino ${ }^{3}$, and GrantJ. Mathews ${ }^{4}$ \\ ${ }^{1}$ College of Bioresource Sciences, Nihon University, Fujisawa 252-8510, Japan \\ ${ }^{2}$ Department of Physics, Soongsil University, Seoul, 156-743, Korea \\ ${ }^{3}$ National Astronomical Observatory of Japan, 2-21-1 Osawa, Mitaka, Tokyo 181-8588, Japan \\ ${ }^{4}$ Center of Astrophysics, Department of Physics, University of Notre Dame, Notre Dame, IN 46556, USA
}

\begin{abstract}
We study pion production from proton synchrotron radiation in the presence of strong magnetic fields by using the exact proton propagator in a strong magnetic field and explicitly including the anomalous magnetic moment. Results in this exact quantum-field approach do not agree with those obtained in the semi-classical approach. Furthermore, we also find that the anomalous magnetic moment of the proton greatly enhances the production rate about by two orders of magnitude, and that the polar angle of an emitted pion is the same as that of an initial proton.
\end{abstract}

\section{Introduction}

Synchrotron radiation can be produced by high-energy protons accelerated in an environment containing a strong magnetic field. This process has been proposed as a source for high-energy photons in the $\mathrm{GeV}-\mathrm{TeV}$ range [1-6]. The meson-nucleon couplings are about 100 times larger than the photon-nucleon coupling, and the meson production process is expected to exceed photon synchrotron emission in the high energy regime.

However, theoretical calculations were performed approximately in a semi-classical way [7-11] and each model gave a different result. In addition, these models could not give a momentumdistribution of a final pion.

In this work we calculate the pionic decay width of proton in the strong magnetic field in the relativistic quantum mechanical framework and compare the results with those in the semi-classical approaches $[10,11]$. Furthermore, we show the energy and angular distributions of emitted pions.

\section{Formalism}

Here, we briefly explain our formalism.

We assume a uniform magnetic field along the $z$-direction, $\boldsymbol{B}=(0,0, B)$, and take the electromagnetic vector potential $A^{\mu}$ to be $A=(0,0, x B, 0)$ at the position $\boldsymbol{r} \equiv g(x, y, z)$. The relativistic

\footnotetext{
ae-mail: maruyama.tomoyuki@nihon-u.ac.jp
} 
proton wave function $\psi$ is obtained from the following Dirac equation:

$$
\left[\alpha_{z} p_{z}-i \alpha_{x} \partial_{x}+\alpha_{y}\left(p_{y}-e B_{x}\right)+M_{N} \beta-\frac{\kappa_{p} e}{m_{N}} B \Sigma_{z}\right] \psi\left(x, p_{z}, s\right)=E \psi\left(x, p_{z}, s\right),
$$

where $M_{N}$ is the proton mass, $\kappa_{p}$ is the proton AMM, $e$ is the elementary charge, and $E$ is the single particle energy written as

$$
E\left(n, p_{z}, s\right)=\sqrt{p_{z}^{2}+\left(\sqrt{2 e B n+M_{N}^{2}}-s \kappa_{p} e B / m_{N}\right)^{2}} .
$$

To calculate the pionic decay width, we use the pseudo-vector coupling interaction Lagrangian density as

$$
\mathcal{L}=\frac{i f_{\pi}}{m_{\pi}} \bar{\psi} \gamma_{5} \gamma_{\mu} \tau_{a} \psi \partial^{\mu} \phi_{a}
$$

where $f_{\pi}$ is the pseudo-vector pion-nucleon coupling constant, $m_{\pi}$ is the pion mass, and $\phi$ is the pion field.

When we use the pseudo-vector coupling for the $\pi N$-interaction, we can obtain the differential decay width of the proton as

$$
\frac{d^{3} \Gamma_{p \pi}}{d q^{3}}=\frac{1}{8 \pi^{2} E_{\pi}}\left(\frac{f_{\pi}}{m_{\pi}}\right)^{2} \sum_{n_{f}, s_{f}} \delta\left(E_{f}+E_{\pi}-E_{i}\right) W_{i f},
$$

with

$$
\begin{aligned}
W_{i f} & =\operatorname{Tr}\left\{\rho_{M}\left(n_{i}, s_{i}, q_{z}\right) O_{\pi} \rho_{M}\left(n_{f}, s_{f}, p_{z}-q_{z}\right) O_{\pi}^{\dagger}\right\}, \\
\rho_{M} & =\frac{\left[E \gamma_{0}+\sqrt{2 e B n} \gamma^{2}-p_{z} \gamma^{3}+M_{N}+\left(\kappa_{p} e B / M_{N}\right) \Sigma_{z}\right]}{4 E}\left[1+\frac{s\left(\kappa_{p} e B / M_{N}+p_{z} \gamma_{5} \gamma^{0}-E \gamma_{5} \gamma^{3}\right)}{\sqrt{\left(2 e B n+M_{N}^{2}\right)}}\right],
\end{aligned}
$$

where $q \equiv\left(q_{0} ; \boldsymbol{q}\right)$ is a momentum of the emitted pion. In the above equation, $f_{n}$ is the harmonic oscillator wave function with the principle quantum number $n$, and

$$
\begin{aligned}
O_{\pi}=\gamma_{5} & \left\{\left[\mathcal{M}\left(n_{i}, n_{f}\right) \frac{1+\Sigma_{z}}{2}+\mathcal{M}\left(n_{i}-1, n_{f}-1\right) \frac{1-\Sigma_{z}}{2}\right]\left[\gamma_{0} q_{0}-\gamma^{3} q_{z}\right]\right. \\
& \left.-\left[\mathcal{M}\left(n_{i}, n_{f}-1\right) \frac{1+\Sigma_{z}}{2}+\mathcal{M}\left(n_{i}-1, n_{f}\right) \frac{1-\Sigma_{z}}{2}\right] \gamma^{2} q_{T}\right\},
\end{aligned}
$$

where $q_{T} \equiv \sqrt{q_{x}^{2}+q_{y}^{2}}$, and $\mathcal{M}\left(n_{1}, n_{2}\right)$ is the harmonic oscillator (HO) overlap function defined as

$$
\mathcal{M}\left(n_{1}, n_{2}\right)=\sqrt{e B} \int d x f_{n_{1}}\left(\sqrt{e B} x+\frac{q_{T}}{2 \sqrt{e B}}\right) f_{n_{2}}\left(\sqrt{e B} x-\frac{q_{T}}{2 \sqrt{e B}}\right) .
$$

\section{Results}

Now, we show numerical results for pion emission.

In Fig.3, we show the total pionic decay widths of the proton with $p_{i z}=0$ and $B=5 \times 10^{17} \mathrm{G}$ as functions of the Landau level $n_{i}$. These decay widths are averaged over the proton spin, $\Gamma=$ 


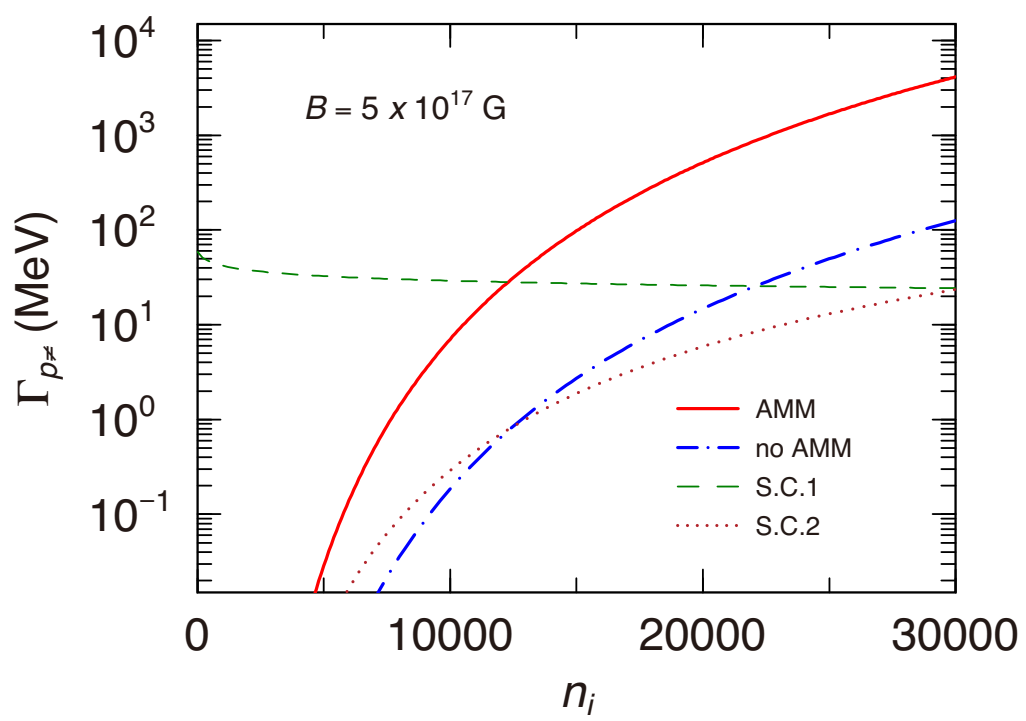

Figure 1. Pionic decay width of protons versus the initial Landau level at $p_{i z}=0$ for $B=5 \times 10^{17} \mathrm{G}$. The decay width is averaged over the proton spin. The solid and dot-dashed lines represent the decay widths of the proton with and without the AMM, respectively. The dashed and dotted lines indicate the results in the semi-classical approaches of Refs. [10] and in [11], respectively.

$\left[\Gamma\left(n_{i},+1\right)+\Gamma\left(n_{i},-1\right)\right] / 2$. The solid and dot-dashed lines represent the decay widths of the proton with and without AMM, respectively.

We see that the decay width with AMM is about 50 times larger than that without AMM. The AMM gives a repulsive potential for $s_{i}=-1$ and attractive for $s_{i}=1$. The transition with $s_{i}=-s_{f}=$ -1 introduces an additional energy to be consumed in the pion production. In addition, the transition with $s_{i}=-s_{f}=1$ reduces the production energy. In this kinematical region a small difference between the initial and final Landau levels changes the transition strength significantly [12]. Therefore, the AMM plays an important role to increase greatly the pionic decay width for $s_{i}=s_{f}=-1$ and to decrease it for $s_{i}=s_{f}=1$.

For comparison, we also plot results in the semi-classical approaches of Ref. [10] (dotted line) and Ref. [11] (dashed line). The results in the semi-classical calculations do not agree with those in the quantum calculation, particularly when the AMM is included.

In order to investigate dominant kinematic conditions, in Fig. 3, we present the decay widths normalized with the total widths when $n_{i}=20000$ and $B=5 \times 10^{17} \mathrm{G}$ as a function of $\left(n_{i}-n_{f}\right) / n_{i}$. The solid and dashed lines represent with the AMM and without AMM, respectively. In all results the initial and final proton spins are set to be $s_{i}=-s_{f}=-1$ because this is the dominant contribution [12].

When comparing the results with and without the AMM, we can see that an AMM effect slightly shifts the peak position to smaller values of $\left(n_{i}-n_{f}\right) / n_{i}$ and broaden the peak width.

Furthermore, we can see that $\left(n_{i}-n_{f}\right) / n_{i} \approx 0.3-0.4$, namely the Landau level difference in the pion emission between the initial and final states is of the same order of the initial and final Landau levels, $\left(n_{i}-n_{f}\right) \sim n_{i} \sim n_{f}$. 


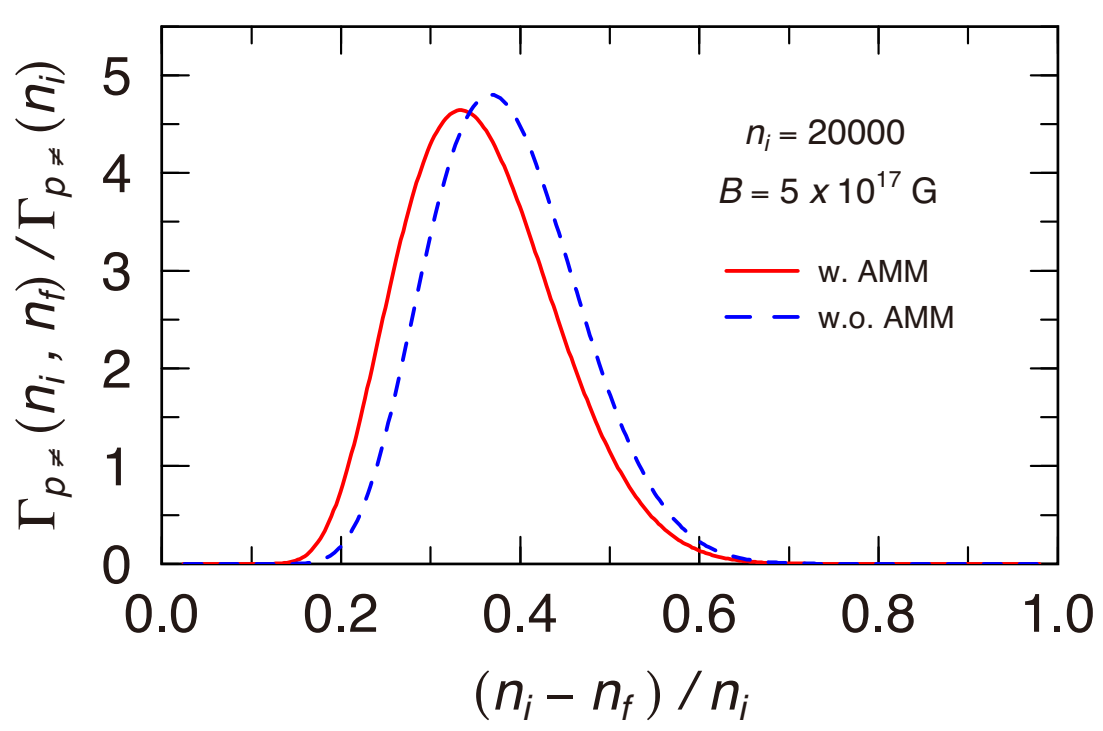

Figure 2. Pionic decay widths of proton normalized with the total decay width when $B=5 \times 10^{17} \mathrm{G}, n_{i}=20000$ and $s_{i}=-s_{f}=-1$ as a function of $\left(n_{i}-n_{f}\right) / n_{i}$. The solid and dashed lines represent the results with and without the AMM, respectively.

In the adiabatic limit it can be assumed that the relative momentum between the final proton and the pion is zero, and that the two particles have the same velocity. In this limit the ratio between these two energies is the same as the mass ratio: $e_{\pi} / e_{f} \approx m_{\pi} / m_{p}$, and the final proton and pion energy become

$$
e_{f} \approx \frac{m_{N}}{m_{N}+m_{\pi}} e_{i}, \quad e_{\pi} \approx \frac{m_{\pi}}{m_{N}+m_{\pi}} e_{i}
$$

The initial proton energy is very large $e_{i} \gg m_{N}, E_{i, f} \approx \sqrt{2 n_{i, f}}$, so that the following relation is given in the adiabatic and high energy limit as $\sqrt{n_{i}}-\sqrt{n_{f}} \approx\left(m_{\pi} / m_{N}\right) \sqrt{n_{i}}$. This leads to

$$
n_{i}-n_{f} \approx \frac{m_{N}^{2}-\left(m_{N}-m_{\pi}\right)^{2}}{m_{N}^{2}} n_{i} \approx 0.28 n_{i} .
$$

The actual $\pi N$-interaction is the $p$-wave, and the relative momentum is not zero even in the adiabatic limit, so that the actual value of $n_{i}-n_{f}$ is larger than the above value; this argument is consistent with the present results.

In the usual semi-classical approximations it is assumed that $n_{i}-n_{v} \ll n_{i}$. This assumption is equivalent to the adiabatic limit when a produced particle is massless such as a photon. Thus, the assumption, $n_{i}-n_{v} \ll n_{i}$, is not satisfied when the emitted particle is massive, so that its results do not agree with the results in the quantum calculations.

In Fig. 3, we show luminosity distribution of the emitted pion, $d^{3} I / d q^{3}=e_{\pi} d^{3} \Gamma / d q^{3}$, with the contour plots, where the energy of the initial proton is $E_{i}-M_{N}=10 \mathrm{GeV}$, the initial spin state $s_{i}=-1$, and the magnetic field $B=5 \times 10^{17} \mathrm{G}$, which corresponds to $n_{i}=n_{\max }=20000$ at $p_{i z}=0$. The luminosities are summarized over the initial Landau levels, $0 \leq n_{\max }-n_{i} \leq 500$, $2500 \leq n_{\max }-n_{i} \leq 3500$ and $5500 \leq n_{\max }-n_{i} \leq 6500$. We see that the pion momentum is distributed narrowly for the polar angle, but is distributed broadly in the absolute value. 
The solid circles indicate the pion momentum in the adiabatic limit, $q_{z}=r_{\pi} p_{i z}$ and $q_{z}=$ $r_{\pi} \sqrt{2 n_{i}+1}$ with $r_{\pi}=m_{\pi} /\left(m_{N}+m_{\pi}\right)$, when $n_{i}=0,3000$ and 6000. We also see that the direction of the emitted pion momentum is very close to that of the initial nucleon momentum.

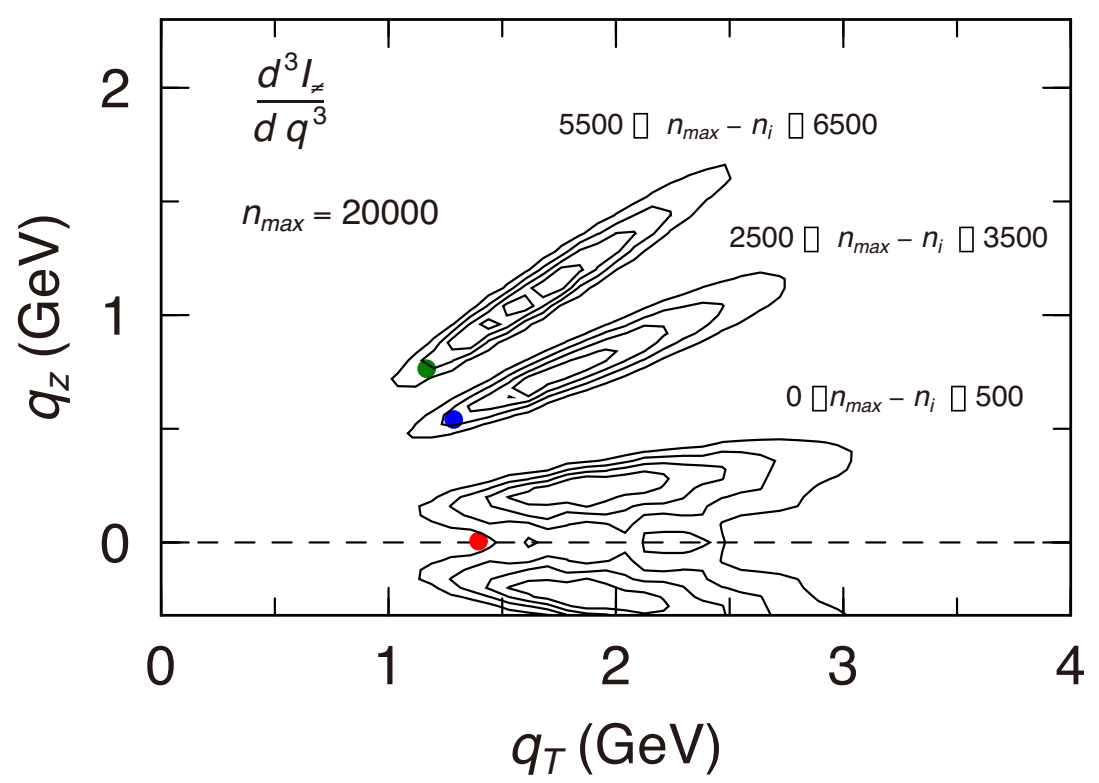

Figure 3. The contour plot of the differential pionic luminosity for the initial proton energy $E_{i}-M_{N}=10 \mathrm{GeV}$, an initial spin state $s_{i}=-1$, and a magnetic field $B=5 \times 10^{17} \mathrm{G}$, where the maximum Landau level is $n_{\max }=20000$. The luminosity is summarized over the initial Landau levels, $0 \leq n_{\max }-n_{i} \leq 500,2500 \leq n_{\max }-n_{i} \leq 3500$ and $5500 \leq n_{\max }-n_{i} \leq 6500$, Each line shows the relative strength. The solid circles indicate the pion momentum in the adiabatic limit. The vertical and horizontal axises are the $z$ component and the transverse component of the emitted pion momentum.

\section{Summary}

we have calculated the pion synchrotron radiation from high energy protons propagating in strong magnetic fields in a microscopic quantum field theoretical framework. We solved the Dirac equation in a strong magnetic field and obtained the proton propagator from its solution. Then, we derived the pionic decay width of the propagating proton in a fully relativistic and quantum mechanical way.

We find out that the anomalous magnetic moment has a very large effect which enlarges the emission rate by about 50 times, and that the polar angles of the emitted pion and the final pion are almost the same as that of the initial proton, when the proton energy is $10 \mathrm{GeV}$ and the magnetic field is $5 \times 10^{17} \mathrm{G}$. One can not know these effects before performing the quantum calculations.

Furthermore, we have shown that the results in our quantum approach do not agree with those in the usual semi-classical approximations. In the usual semi-classical approximation it is assumed that $n_{i}-n_{v} \ll n_{i}$, where the $\mathrm{HO}$ overlap function $\mathcal{M}$ can be approximately written with the Airy function. This assumption is not satisfied when a produced particle is massive, 
In actual magnetars the surface magnetic field is known to be of order $B \sim 10^{15} \mathrm{G}$. In the present method we did not perform a calculation for such a magnetic field strength because of the large number of Landau levels involved: $n_{i} \sim 10^{12}-10^{13}$. As for future studies, we must consider a method to treat huge numbers of the Landau levels for the magnetic field of $B \sim 10^{15} \mathrm{G}$.

\section{References}

[1] N. Gupta and B. Zhang, MNRAS, 380, 78 (2007).

[2] M. Böttcher, C. D. Dermer, ApJL, 499, L131 (1998).

[3] T. Totani, ApJL, 502, L13 (1998).

[4] P.C. Fragile, G.J. Mathews, J. Poirier and T. Totani, APh 20, 591 (2004).

[5] K. Asano and S. Inoue, ApJ, 671, 645 (2007) .

[6] K. Asano, S. Inoue and P. Mészáros, ApJ, 699, 953 (2009).

[7] V.L. Ginzburg and S.I. Syrovatskii, UsFiN, 87, 65 (1965).

[8] V.L. Ginzburg and S.I. Syrovatskii, Annu. Rev. Astron. Astrophys. 3, 297 (1965).

[9] G.F. Zharkov, Sov. J. Nucl. Phys., 1, 17314 (1965).

[10] A. Tokushita and T. Kajino, Astrophys. J. 525, L117 (1999).

[11] V. Berezinsky, A. Dolgoy and M. Kachelriess, Phys. Lett. B 351, 261 (1995)

[12] T. Maruyama, M.-K. Cheoun, T. Kajino Y. Kwon, G.J. Mathews, Phys. Rev. D91,123007 (2015) 